\title{
Magnetic wood by in situ synthesis of iron oxide nanoparticles via a microwave-assisted route
}

\author{
Jana S. Segmehl* a,b, Anna Laromainec, Tobias Keplinger*a,b, Anna May- \\ Masnou $^{c}$, Ingo Burgert ${ }^{a, b}$ and Anna Roig ${ }^{c}$
}

a.

Wood Materials Science, Institute for Building Materials (IfB), ETH Zürich, Stefano Franscini-Platz 3, 8093

Zürich, Switzerland

E-mail: tkeplinger@ethz.ch, jana@segehl-energie.de

b. Applied Wood Materials Laboratory, EMPA-Swiss Federal Laboratories for Materials Science and Technology, 8600 Dübendorf, Switzerland

c. Institut de Ciència de Materials de Barcelona (ICMAB), Campus UAB, Bellaterra, Catalonia, E-08193, Spain

Functional materials with high porosity and hierarchical structure are highly demanded for numerous material applications. In this study a magnetic hybrid material derived from wood and superparamagnetic iron oxide nanoparticles (SPIONs), was synthesized by microwave-assisted thermal decomposition. This novel in situ functionalization approach resulted in a homogeneous distribution of the integrated inorganic component within the entire complex wood cell wall structure, which was previously not achieved. In a detailed investigation based on confocal Raman microscopy imaging, transmission electron microscopy and optical microscopy the precipitated phase and the resulting hybrid structure were characterized. Magnetic measurements revealed the impact of the anisotropic wood scaffold on the integrated magnetic functionality and confirmed the isotropic superparamagnetic characteristics of the in situ precipitated nanoparticles. Therewith, it is clearly demonstrated, that the anisotropic properties of the obtained hybrid material result from the particle organization in the given spruce wood structure and no alteration of particle properties is induced by the presence of the lignocellulosic material.

This document is the accepted manuscript version of the following article: Segmeh1, J. S., Laromaine, A., Keplinger, T., May-Masnou, A., Burgert, I., \& Roig, A. (2018). Magnetic wood by in situ synthesis of iron oxide nanoparticles via a microwave-assisted route. Journal of Materials chemistry $c, 6(13)$, 3395-3402. https://doi.org/10.1039/c7tc05849g 


\section{Introduction}

Among the broad spectra of biological materials, the advantageous combination of inorganic and organic entities organized in a hierarchical architecture represents a common design principle. $\underline{1}$ This concept of hybridization found in nature has been successfully adapted in material science for the development of novel light weight hierarchical material systems.1-3 Such three dimensional functional porous materials are highly demanded in material science and engineering for low density applications, e.g. as capacitors, in batteries and as storage devices.2-4 However, it still remains a challenge to reach the same level of complex structuring of biological materials by synthetic approaches. $\underline{5}$ Therefore, the functionalization of natural materials (e.g. wood) is a promising alternative, as it allows for directly profiting from its inherit architecture. In this regard numerous functionalization approaches for wood, targeting the combination of its beneficial intrinsic properties (e.g. outstanding mechanics at low density) and new functionalities (e.g. magnetic properties, conductivity, stimuli responsiveness and transparency) have been developed within the last years. Thereby, different types of functional materials including polymers and minerals have been incorporated or in situ formed in the wood structure. $\underline{6-17}$

In order to retain the low density of the wood based hybrid material and to obtain strong functionality, a sufficient and controllable functionalization of wood cell walls is needed. A common limitation of previously reported approaches on the formation of inorganic phases within wood is an increased density due to considerable material deposition within the cell lumen and, in consequence, a low degree of additional functionality within the cell walls.17-19 To circumvent this limitation and increase the concentration of material deposited in the cell wall structure, processes to increase the porosity of the initial structure (e.g. delignification) or approaches to integrate the secondary material via in situ precipitation have to be implied. $\underline{8}$ However, considerable delignification often results in a deterioration of the mechanical properties and structural integrity of the samples whereas in situ approaches allow to mostly retain the structural assembly. Thus, new synthesis approaches allowing for a homogeneous and efficient deposition of inorganic particles within the cell walls are required.

Here we propose the application of microwave-assisted formation of metal oxide nanoparticles via a non-aqueous thermal decomposition process as a novel functionalization approach for wood cell walls (Fig. 1). The synthesis of metal oxide nanoparticles by a non-aqueous sol-gel process, thermally activated by classical heating or microwave assisted, was shown to be a highly versatile route for the preparation of various, even complex oxide nanoparticle systems with good control over their properties.20 Further, microwave-assisted processes were favoured in the last years, as the energy consumption during processing is significantly reduced. The volumetric heating of the solvent in the microwave diminishes temperature gradients typical for hot plate heating and the synthesis of highly monodisperse nanoparticles can be reached in significantly reduced reaction times. $21, \underline{22}$ In our study, we adapted the synthesis of superparamagnetic iron oxide nanoparticles (SPIONs) to functionalize wood cell walls, in order to control the precipitated phase and add functionality. $\underline{23}$ The even temperature profile and the wood inherent polar 


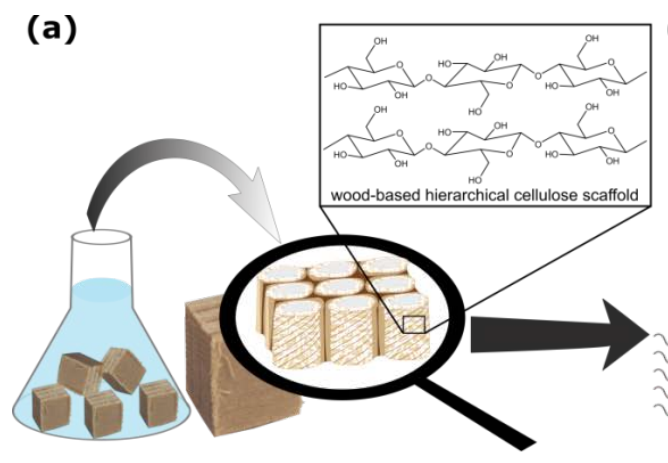

(b)

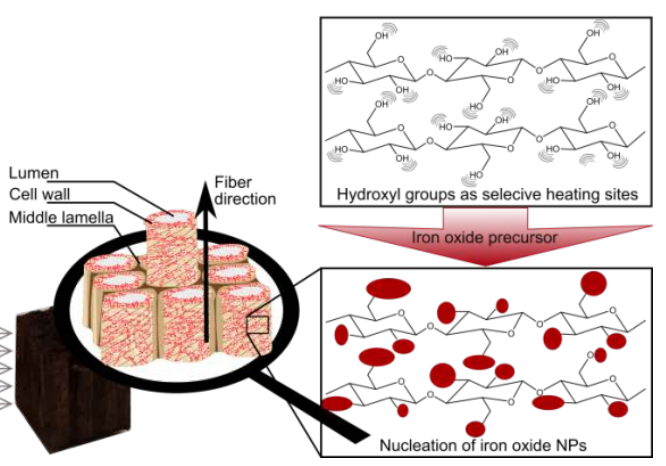

Fig. 1 Scheme of the functionalization approach. (a) pretreatment of the wood cubes in $\mathrm{NaOH}$ solution to enhance the porosity prior to particle synthesis. (b) microwave assisted functionalization with SPIONs for magnetic properties using the wood inherent hydroxyl groups as selective heating sites

hydroxyl groups, which act as hot spots to induce a synchronic nucleation, allow for a homogeneous particle distribution and final functionality.

Potential applications of magnetically functionalized wood specimens include electromagnetic shielding or actuation and induction heating as previously proposed.24-28 Yet, even besides technology driven applications, the hierarchical porous structure provided by wood, is a feasible experimental platform to unravel aspects of the process for particle formation, like precursor diffusion, local temperature distribution and the nature of interfaces between cellulose and inorganic structures.

\section{Experimental}

\section{Preparation of wood samples}

Small cubes $(5 \times 5 \times 5 \mathrm{~mm} 3)$ of Norway spruce (Picea abies), cut with a circular saw, were functionalized by in situ synthesis of SPIONs. To enhance the porosity prior to the synthesis, the samples were treated for $6 \mathrm{~h}$ at $80{ }^{\circ} \mathrm{C}$ in a sodium hydroxide (Sigma Aldrich) solution (10 wt.\%). An extensive washing with Milli-Q water and a final solvent exchange to ethanol completed the pretreatment.

Microwave assisted synthesis: Precipitation of superparamagnetic iron oxide nanoparticles (SPIONs) in wood

For the in situ synthesis of iron oxide nanoparticles (SPIONs) within wood cell walls, a procedure previously established by Zeng et al. on bacteria cellulose films was used.23 In short, $0.35 \mathrm{mmol}$ (123.6 mg) iron (III) acetylacetonate (Fe(acac) , $97 \%$, Sigma-Aldrich) as the iron precursor was dissolved in $4.5 \mathrm{~mL}$ benzyl alcohol ( $\mathrm{BnOH}$, Sigma-Aldrich) and the wood cubes were immersed in this solution prior to the synthesis. The incubation time was varied between 12 hours and 6 days. 
The microwave-assisted synthesis was carried out in a CEM Discover reactor (Explorer 12-Hybrid) operating at a frequency of $2.45 \mathrm{GHz}$ and an automated power control based on a temperature feedback with a maximum power of $300 \mathrm{~W}$. The microwave tubes containing the precursor solution and the samples were preheated to $60^{\circ} \mathrm{C}$ for $12 \mathrm{~min}$ for equilibration before increasing the reaction temperature, at which the sample was kept for 20 min before the solution was automatically cooled down to $50^{\circ} \mathrm{C}$. Three different reaction temperatures were chosen $\left(120^{\circ} \mathrm{C}, 160^{\circ} \mathrm{C}\right.$ and $\left.200^{\circ} \mathrm{C}\right)$ to investigate the influence on the formed SPIONs and the obtained hybrid material. After the synthesis, the functionalized wood samples were removed and placed in an acetone bath and sonicated for $3 \mathrm{~min}$ for cleaning. This cleaning step was repeated until the solution remained clear after sonication. Then samples were dried in a vacuum oven at $70^{\circ} \mathrm{C}$ for 2 days prior to further investigations.

SPIONs in solution were collected by adding $40 \mathrm{~mL}$ acetone with $20 \mu \mathrm{L}$ trimethyl ammonium hydroxide (TMAOH, $25 \%$, Sigma-Aldrich) (used as electrostatic surfactant) and centrifuged at $6000 \mathrm{rpm}$ for $20 \mathrm{~min}$. The supernatant from the first centrifugation was discarded and the precipitate was re-dispersed in the same amount of acetone with $\mathrm{TMAOH}$ and centrifuged again. The final precipitate was dried overnight in an oven at $70^{\circ} \mathrm{C}$ and was redispersed in $2 \mathrm{~mL}$ deionized water containing $10 \mu \mathrm{LTMAOH}$ to yield a time stable dispersion.

\section{Characterization techniques}

Optical microscopy and scanning electron microscopy (SEM). Longitudinal cuts and block faces in transverse direction were prepared with a rotary microtome from the center of the modified specimen for investigations by optical microscopy in reflection and scanning electron microscopy in backscattered configuration. Optical microscopy was conducted using an OLYMPUS BX51 microscope and SEM investigations were performed with a QUANTA FEI200 FEG-ESEM under low vacuum conditions, an acceleration voltage of $20 \mathrm{kV}$ and a working distance of $7 \mathrm{~mm}$.

Transmission electron microscopy (TEM). Ultrathin wood chips $(0.25 \mu \mathrm{m})$ of the modified wood samples were cleaved from the sample center using a rotary microtome (Leica, RM 2255 Germany) equipped with a glass knife. SPIONs samples were prepared by dripping a drop of the particle solution on a TEM grid and waiting for evaporation of the solvent. TEM images were obtained with a JEOL JEM-1210 electron microscope, operating at $120 \mathrm{kV}$. The NPs mean size was computed from 200 nanoparticles and the size histogram fitted to a Gaussian function.

Dynamic Light Scattering (DLS). DLS was used to determine the average hydrodynamic diameter of SPIONs. The measurement was conducted with a Zetasizer Nano ZS (Malvern Instruments, Germany) equipped with a He/Ne $633 \mathrm{~nm}$ laser, and the SPIONS solutions were placed in PMMA cuvettes. The stock solution was diluted to a concentration of $100 \mu \mathrm{L} / \mathrm{mL}$ in Milli-Q water. Data analysis was conducted with the measurement software (Malvern Instruments, Germany). 
Thermogravimetric analysis (TGA). Fine powder of the dry samples was prepared using a rotary microtome. Between $2-3 \mathrm{mg}$ of the powder were used for the thermogravimetric analysis. Measurements were performed using a TGA-DSC/DTA analyzer (TA Instruments, Q50) with a heating rate of $10^{\circ} \mathrm{C}$ min- 1 from RT to $800{ }^{\circ} \mathrm{C}$ in air.

Confocal Raman microscopy. Confocal Raman microscopy imaging was conducted on thin sections prepared from the inside of the functionalized wood samples. The samples were sealed in Milli-Q water between a glass slide and a cover slip $(0.17 \mathrm{~mm})$. All measurements were recorded in backscattering configuration with an InVia Raman microscope (Renishaw, UK) equipped with a motorized xyz-stage for mapping. The excitation of Raman Scattering was operated with a linearly polarized He/Ne-laser $(\lambda=633 \mathrm{~nm})$. A 100 x oil immersion objective with numerical aperture (NA) 1.3 (Nikon) was used. The Raman signal was detected by an air cooled charge coupled device (CCD) camera behind a spectrometer (InVia) with a spectral resolution of about $1 \mathrm{~cm}-1$. The mapping was recorded with an integration time of $4 \mathrm{~s}$ and a laser power of $18 \mathrm{~mW}$ in the spectral region between $250-3100 \mathrm{~cm}-1$ and a step size of $500 \mathrm{~nm}$. The measurement setup as well as the initial data treatment, including cosmic ray removal and baseline correction, was done in Wire (version 4.1, Renishaw, UK). For baseline correction, the inbuilt automatic intelligent background removal was used. The data was further processed in the Matlab based software Cytospec (version 2.00.01). Regions of interest (ROI) from the secondary cell wall and the cell corner area of each measured mapping were extracted and averages were calculated. All average spectra were min-max normalized on the band at $380 \mathrm{~cm}-1$ using OPUS (version 7.2, Bruker, Germany).

Magnetic measurements. Magnetic properties of the samples were characterized with a superconducting quantum interference device (SQUID) magnetometer (Quantum Design MPMS5XL), comprising the measurement of the magnetization versus the applied magnetic field at constant temperature $(\mathrm{M}(\mathrm{H})$ curves at $300 \mathrm{~K}$ and $5 \mathrm{~K})$ and magnetization versus the temperature at a constant applied field (ZFC-FC curves at 50 Oe).

\section{Results and discussion}

The preparation of the magnetically functionalized wood was conducted via an in situ microwave-assisted synthesis of SPIONs.23 Previously used for the functionalization of bacteria cellulose films, the procedure was adapted for the three-dimensional architecture of wood. Incubation time in the precursor solution, synthesis temperature, and equilibration time in the microwave were optimized in order to enhance the efficiency of the functionalization procedure.

A first visual comparison of the specimens functionalized under different process conditions revealed a substantial darkening of the 


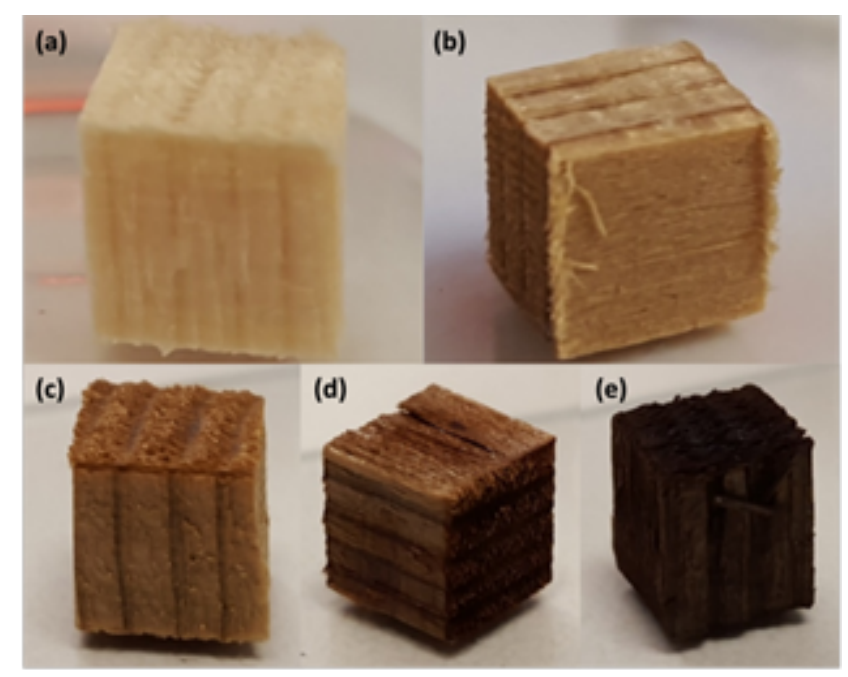

Fig. 2 Pictures of native wood samples $\left(5 \times 5 \times 5 \mathrm{~mm}^{3}\right)$ functionalized with iron oxide nanoparticles using the microwave assisted synthesis procedure (a) native wood sample, untreated; (b) wood sample in $\mathrm{BnOH}$ processed in the microwave at $200^{\circ} \mathrm{C}$, without the addition of the iron precursor; (c) wood sample after 6 days incubation in precursor solution and microwave process at $120^{\circ} \mathrm{C}$; (d) wood sample after 6 days incubation in precursor solution and microwave process at $160^{\circ} \mathrm{C}$; (e) wood sample after 6 days incubation in precursor solution and microwave process at $200^{\circ} \mathrm{C}$

samples with increasing process temperature and incubation time, while equilibration time in the microwave did not influence the obtained material (Fig. 2). Samples prepared at $200^{\circ} \mathrm{C}$ (Fig. 2e) showed a dark brownish color and the difference in this darkening was only minor between short (12 hours) and long (6 days) incubation times. Samples processed at $160{ }^{\circ} \mathrm{C}$ and $120^{\circ} \mathrm{C}$ were brighter and of a more reddish brown color (Fig. 2c,d) than samples processed at $200^{\circ} \mathrm{C}$. These results indicate that a complete transformation of the precursor requires a process temperature above $160{ }^{\circ} \mathrm{C}$. This is supported by a subsequent thermogravimetric analysis, where the obtained residual mass can be assigned to absolute SPIONs content in the samples. For the modified samples functionalized at $200{ }^{\circ} \mathrm{C}$ after 6 days of incubation time, a residual mass of $4.8 \mathrm{w} \%$ was measured, whereas the residual mass of samples synthesized at $120^{\circ} \mathrm{C}$ was $3.9 \mathrm{w} \%$. In addition to the darkening of the material due to functionalization, a correlated variation in mechanical properties was further observed. While the reference material showed a higher brittleness during cutting than native wood, the functionalization resulted in a material with higher mechanical resistance during cutting in comparison to unmodified reference samples. Therefore, some reinforcement of the wooden 


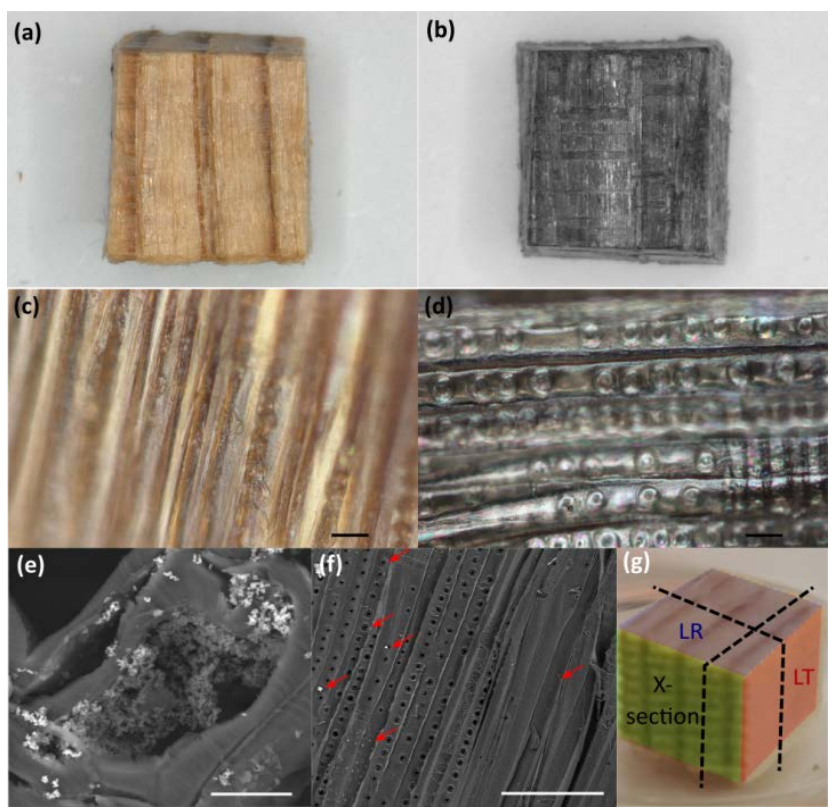

Fig. 3 Microscopic investigations. Optical microscopy of entire LR planes and longitudinal cuts (LT) (a-d) prepared from the center of the cubic samples. $(a, c)$ Reference sample: wood treated in the microwave in benzyl alcohol without the addition of the iron precursor. (b,d) Sample incubated for 6 days in the iron precursor solution and microwave treated at $200{ }^{\circ} \mathrm{C}$. A metallization of the inner surface of the cube can be observed in clear contrast to the sample solely processed in benzyl alcohol. (e) Scanning electron microscopy in backscattering configuration on prepared cross sections revealed the presence of particle agglomerates and clusters in the lumen structure and their scarce and random distribution was observed on longitudinal cuts (LR) ( $f$, red arrows). (g) Schematic indicating the three different cut directions longitudinal radial (LR), longitudinal tangential (LT) and cross section (X-section). (c,d) Scale bar $=30 \mu \mathrm{m}$. (e) Scale bar $=10 \mu \mathrm{m}$. (f) Scale bar $=200 \mu \mathrm{m}$.

structure can be achieved due to the iron oxide precipitation.

To gain more detailed information on the distribution of the formed particles in the wood samples, optical microscopy on longitudinal block faces, prepared by cleavage of the modified cubes through their center, was conducted (Fig. 3a-d). Although no particles or agglomerates were identified on the inner surfaces of the lumen, a strong grey metallic colorization (Fig. 3b,d) was observed in contrast to the beige coloration of only solvent treated samples (Fig. 3a,c). This homogeneous darkening and metallization of the samples proves a homogeneous distribution of the precipitated material through the whole sample volume.

Based on the observations in optical microscopy a homogeneously distributed layer of nanoparticles was expected to be visualized with scanning electron microscopy (SEM) on the lumen cell wall interface. The presence of agglomerates and clusters of smaller particles were identified (Fig. 3e,f). The apparent absence of a dense particle layer at the interface region in combination with the metallic colorization throughout the whole wood sample (Fig. 3b,d) points towards a homogeneous dispersion of ultra-small nanoparticles in the organic scaffold, which cannot 
be resolved due to the limited magnification of electron microscopy in the presence of the organic lignocellulosic material.

For a detailed understanding of the particle deposition and the influence of the various process parameters on particle formation, SPIONs formed during the synthesis in the supernatant and in the wood structure were independently investigated. For the particles formed within the wood samples, TEM measurements were conducted on ultrathin chips, which were cleaved from the inner part of the functionalized specimens using a microtome and deposited on TEM grids. The ultrathin slices from the center of the cubes presented the same dark homogeneous coloration as seen in Figure 1e. The particles found in the supernatant were also observed by TEM. Size analysis histograms revealed no correlation between size distribution of particles in the supernatant and incubation time (Fig. 4a,e). Yet, a distinct dependence of the particle size on reaction temperature was observed. For the samples processed at $200{ }^{\circ} \mathrm{C}$, an average size of $6.7 \mathrm{~nm}$ was determined at short incubation times, while the longest incubation time resulted in an average size of $7.3 \mathrm{~nm}$ (Fig. 4a). The supernatant of samples processed at 160 ${ }^{\circ} \mathrm{C}$ revealed a slightly bigger average size of $9.3 \mathrm{~nm}$ for particles found in solution, whereas in the supernatant retained from the synthesis at the lowest process temperature of $120^{\circ} \mathrm{C}$, no particles were found. In consequence, for a transformation of the precursor molecules into stable nanoparticles through thermally activated oxidative decomposition, a minimal temperature of $160^{\circ} \mathrm{C}$ was necessary as at $120^{\circ} \mathrm{C}$, the activation energy is not sufficient to result in an efficient material precipitation.

A decoration of the lignocellulosic material with small nanoparticles could be visualized by TEM investigations (Fig. 4b). The detailed analysis revealed differences between the deposited material depending on the synthesis parameters, but also between particles formed in solution and in close association to the lignocellulosic scaffold. The statistical evaluation of the average size of particles formed in close association to the lignocellulosic material showed a smaller average size $(5.2 \mathrm{~nm} \pm 1.4)$ than for the SPIONs in solution ( $7.3 \mathrm{~nm} \pm 1.7)$ (Fig. 4c). This is further supported by the TEM image, containing both, particles formed in the scaffold (Fig. 4b, blue area) and free particles that remained in loose vicinity but were not attached to the structure (Fig. $4 \mathrm{~b}$, red area). The small confinement due to the internal porosity present in the cell wall structure, variations in the concentration of particle nucleation sites inside and outside of the wood structure, and differences in the precursor concentration between the scaffold structure and the surrounding liquid phase are possible reasons. The particles observed in the modified wood material were predominant of rough spheroidal morphology and the ring pattern obtained by electron diffraction, which was assigned to the spinel structure of maghemite, confirmed $\mathrm{y}$-Fe $\mathrm{O}_{3}$ as the predominant iron oxide phase present (Fig. 4d). 29 The large hydrodynamic radius observed with DLS for the supernatant synthesized at $120^{\circ} \mathrm{C}$ is a strong indication for the incomplete transformation of the precursor and the presence of intermediate reaction products (Fig. 4e).

For a definite proof of the formation of nano-sized particles within the wood cell wall structure confocal Raman spectroscopy imaging was used as the high spatial and chemical resolution of this technique is required to 
determine and identify the inorganic phase. Raman maps across double cell walls were acquired on thin sections from the inside of the functionalized wood samples (Fig. 5). A deposition of iron oxide particles within the whole secondary cell wall including also the compound middle lamella region could be confirmed (Fig. 5b,c).

This uniform deposition of nanoparticles throughout the secondary cell wall and compound middle lamella, is with high probability enabled by the small size of the iron precursor, allowing a diffusion driven access to the wood cell wall structure. Such an infiltration of wood cell walls can either take place via the lumen or as Fengel et al observed for the impregnation with metal salt solutions, along the interconnected middle lamellae and cell corners region. $\underline{30}$ Based on our results, it is reasonable to conclude that both transport pathways, are of relevance for this type of functionalization.

While the main features in the average spectra of the cell wall are assigned to the organic wood inherit constituents, mainly cellulose and lignin, additional spectral contributions characteristic for iron oxide morphologies were detected in the low frequency range $(150-800 \mathrm{~cm}-1) .31$, 32Depending on the iron oxide morphology present in the wood cell walls, different characteristic bands appear as indicators of the specific iron oxide phase within the system. Based on previous investigations and literature data on the applied synthesis, magnetite and maghemite, with minor contribution of hematite were the main morphologies to be expected.23, $\underline{29}$ The spectral region between $500-700 \mathrm{~cm}-1$ in the Raman spectra of iron oxide functionalized wood samples is characterized by a set of overlapping spectral features, dominated by a broad maxima 


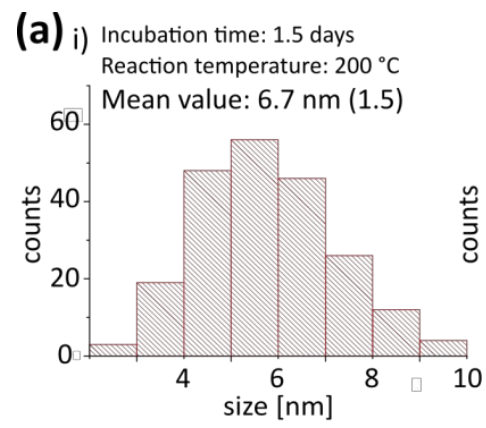

ii) Incubation time: 6 days
Reaction temperature: $200{ }^{\circ} \mathrm{C}$
Mean value: $7.3 \mathrm{~nm}(1.7)$
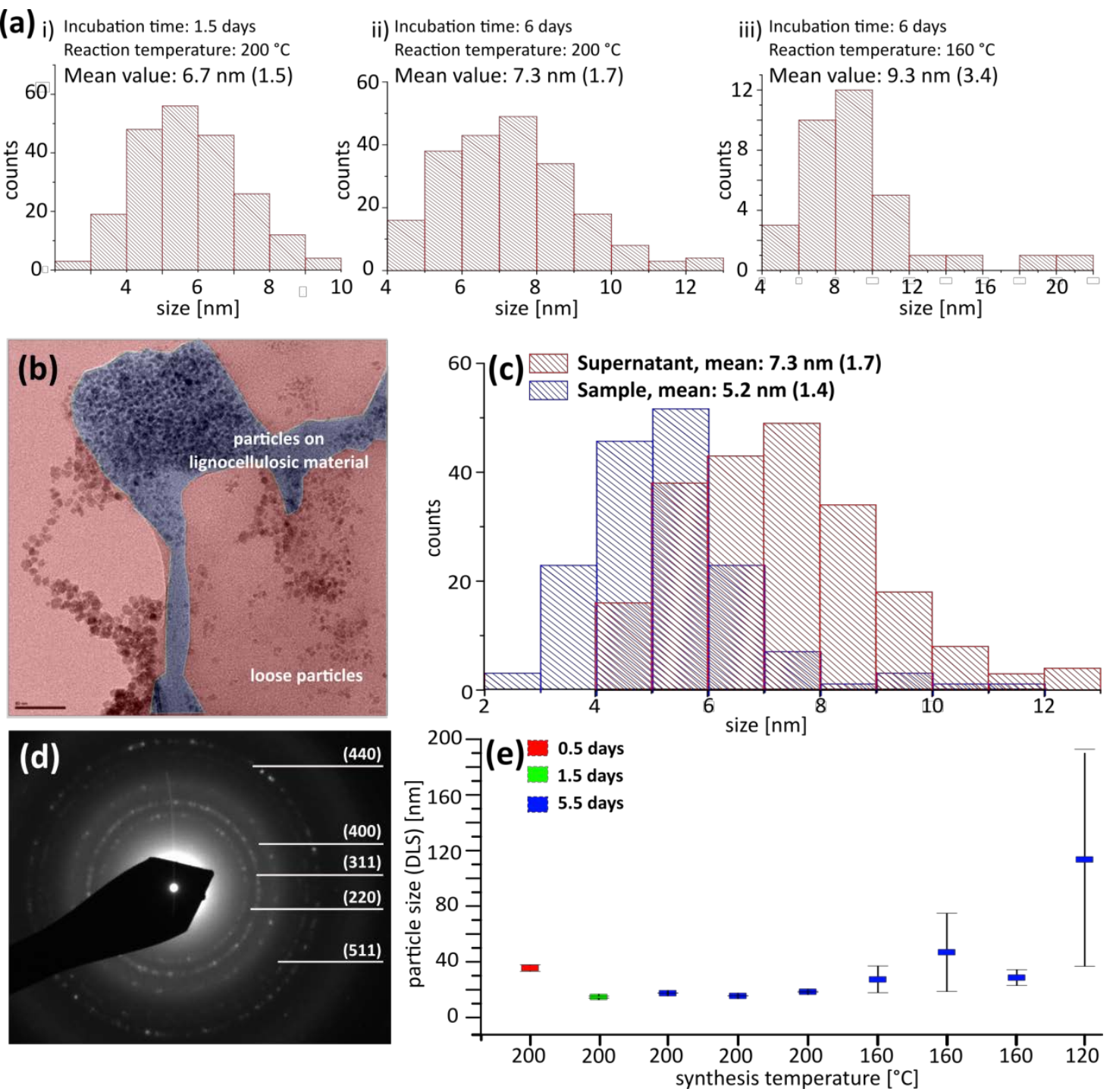

Fig. 4 (a) Particle size distribution based on statistical analysis of TEM images. Size distribution for particles found in the supernatant after functionalization. Synthesis parameters: i) $36 \mathrm{~h}$ incubation time, $200{ }^{\circ} \mathrm{C}$ process temperature, ii) 6 days incubation time, $200{ }^{\circ} \mathrm{C}$ process temperature, and iii) 6 days incubation time, $160^{\circ} \mathrm{C}$ process temperature. (b) Image of iron oxide nanoparticles, found on (blue area) and in close association (red area) to the modified lignocellulosic material. Scale bar $=50 \mathrm{~nm}$. (c) Statistical analysis of the particle size for SPIONs formed in the lignocellulosic material (blue) and in the supernatant (red). The TEM based analysis revealed differences in the size distribution. Particles formed in the scaffold were smaller than particles found in close association. Mean value and standard deviation (in parenthesis) were computed. (d) Electron diffraction pattern of super paramagnetic iron oxide nanoparticles, formed in the wood derived scaffold. (e) DLS based analysis of the hydrodynamic radius for particles found in the supernatant of the synthesis. Independent of the incubation time ( 0.5 days = red, 1.5 days = green, 5.5 days = blue), particles formed at $200{ }^{\circ} \mathrm{C}$ were smaller than particles formed at $160{ }^{\circ} \mathrm{C}$ and below. The distribution of the hydrodynamic radius was broad for low synthesis temperature and narrow for particles found after the synthesis at $200^{\circ} \mathrm{C}$. 

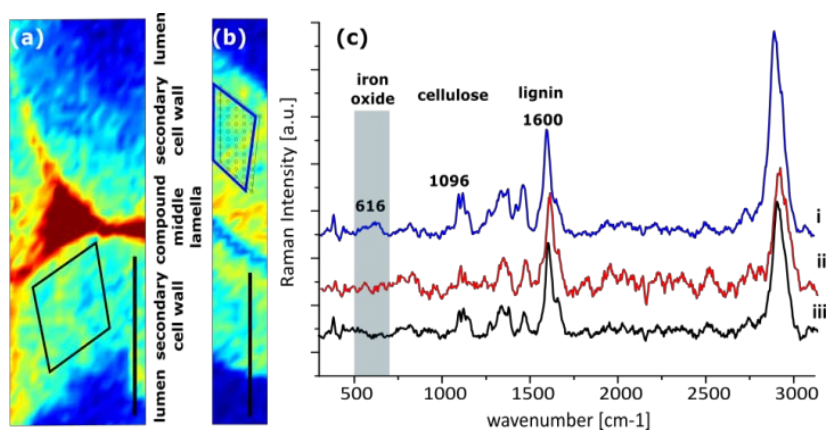

Fig. 5 Confocal Raman microscopy on iron oxide functionalized wood samples. Integration image visualizing the cell wall according to lignin distribution (1540-1680 $\mathrm{cm}^{-1}$ ) in (a) wood sample treated in the microwave in benzyl alcohol without the addition of the iron precursor and (b) wood sample treated in the microwave in benzyl alcohol with addition of the iron precursor. (c) A comparison of the obtained average spectra extracted from the secondary cell wall in the respective mapping (marked areas in (a) and (b)) verifies the presence of iron oxide in this area. i) average spectra extracted from the functionalized sample in b); ii) reference spectra for the prepared wood sample without functionalization (a), extracted average from measurement displayed in (a); iii) reference spectra of native spruce wood. Scale bar $=10 \mu \mathrm{m}$

around $616 \mathrm{~cm}-1$ (highlighted in figure 4c). Although this main peak around $616 \mathrm{~cm}-1$ is in close vicinity to an E mode reported for hematite, a contribution of magnetite $\left(\mathrm{A}_{1 \mathrm{~g}}\right.$ and $\mathrm{T}_{2 \mathrm{~g}}$ mode at $662 \mathrm{~cm}-1$ and $538 \mathrm{~cm}-1$, respectively) and maghemite (two broad bands around 500 and $660-720 \mathrm{~cm}-1$ ), to the observed set of overlapping bands can be confirmed.31-36 Therefore, the iron oxide phase deposited in the wood structure consists of a mixture of hematite, magnetite and maghemite. While magnetite and maghemite are morphologies typically reported for the applied synthesis and confirmed with electron diffraction as well as by the dark brown color of the macroscopic samples, the presence of hematite was not expected.23, 26-29 Strong auto-fluorescence of the wooden substrate as result of the preparative treatment required high laser power and long integration time to acquire the Raman spectra. Thus, a laser induced oxidative degradation of magnetite and maghemite to hematite seems to be likely and could not be circumvented. Such structural transformation due to intense laser irradiation was previously reported and is further supported by the observed broad spectral features and a slight shift to lower wavenumbers compared to the sharp characteristic signature reported for synthesized hematite material. $\underline{31}, \underline{33}, \underline{34}$

To study the magnetic properties of the iron oxide functionalized samples, magnetic measurements were performed using a SQUID magnetometer. The characteristic dependencies in magnetization versus applied magnetic field at constant temperature and magnetization versus temperature at applied constant field were evaluated (Fig.6). The lack of remanence and coercivity of the magnetization in dependence of the applied 
magnetic field, evidence the superparamagnetic properties of the particles (Fig. 6a). Measurements with the magnetic field applied parallel to the longitudinal and transverse directions of the sample did not result in a detectable anisotropy, which is in contrast to previous reports on magnetized wooden structures, in which an anisotropic behavior was observed in relation to the main directions in the wooden material. $\underline{12}, \underline{17}$ Therefore, it is concluded that the procedure for the formation of SPIONs within wood cells applied in this study resulted in a homogeneous distribution in all spatial directions with no preferential deposition and elongation of particles along the cellulose fibers. Contrary, in the previously reported systems, a heterogeneous distribution of the magnetic deposits on the cell wall level was prevailing and comparatively large clusters were formed. Field cooled-zero field cooled (FC-ZFC) curves displaying the magnetization under constant small field and in dependence of the temperature, confirm the room temperature superparamagnetic nature of the internalized particles (Fig. 6a, inset). The maximum of the ZFC curve signals the blocking temperature $(T=36 \mathrm{~K})$ that confirms the particles ultra-small size. Further, the sharpness of the ZFC curves indicates that the particles present in the scaffold are of narrow size distribution and therewith support the TEM results (Fig. 3). The increase of the FC curve at low 

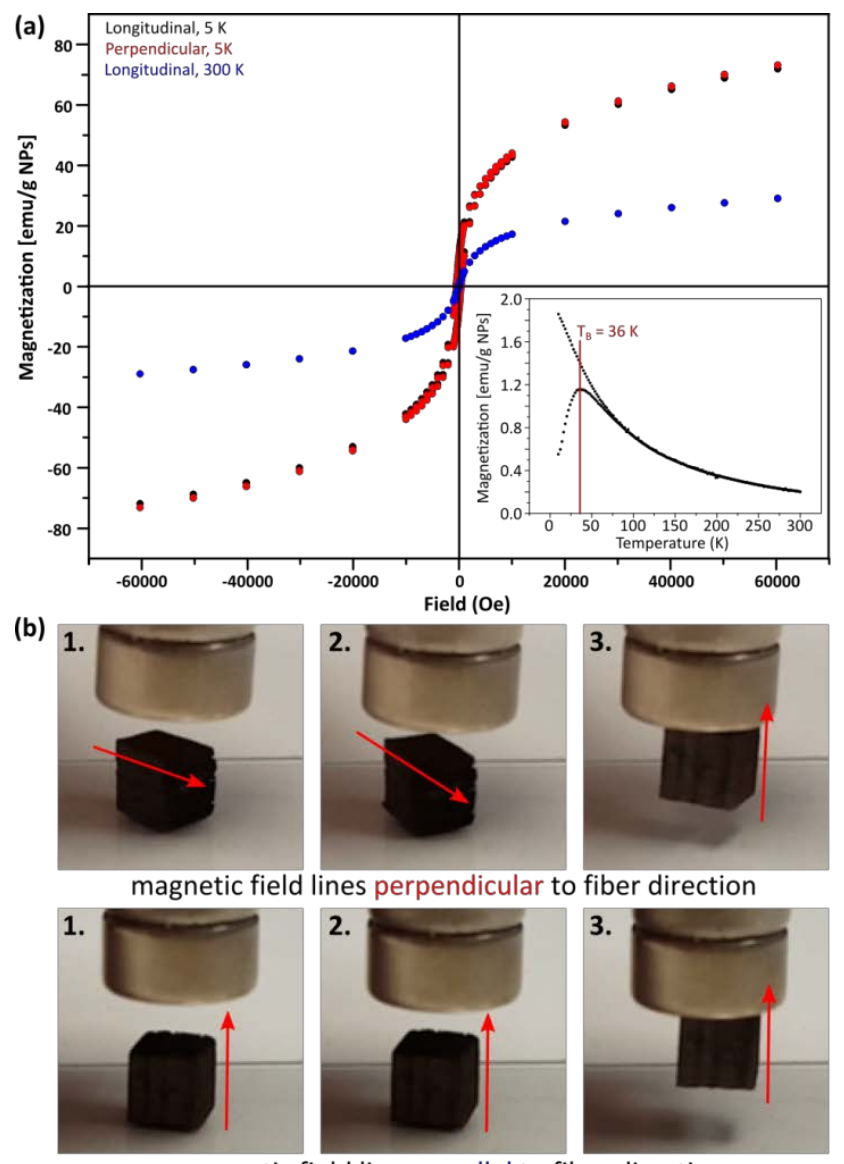

magnetic field lines parallel to fiber direction

Fig. 6 Magnetic measurements of the iron oxide functionalized wooden structure. (a) Magnetization versus magnetic field. The typical features for superparamagnetic material, e.g. lack of remanence and coercitivity, confirm the superparamagnetic properties of the induced particles. Inset: FC-ZFC measurement. The low blocking temperature indicates a small domain size in the formed material. (b) Sequential pictures taken from a video, showing the anisotropy of the response of the macroscopic sample to an external magnetic field. Arrows indicate fiber direction.

temperatures and the positive slope of $\mathrm{M}(\mathrm{H})$ at high fields point towards the presence of additional paramagnetic compounds, probably Fe3+ ions in the sample.

In comparison to previously reported magnetized wooden structures, where the anisotropic behavior, correlated with the main directions in the wooden material and a heterogeneous distribution of magnetic deposits was observed $\underline{12}, \underline{17}, \underline{28}$, the here applied procedure resulted in a fine dispersed secondary phase of the ultra-small isotropic particles. A complete infiltration of the wood structure with the precursor and subsequent homogeneous distribution of the deposited magnetic phase throughout all anatomical regions was established. No preference in growth direction for the deposited material, neither anisotropy in the shape of the particles, nor a correlation between particle distribution and underlying cellulosic scaffold was apparent. This difference in morphology and microscopic distribution of the magnetic deposits to previously reported magnetized wood structures with an 
anisotropic magnetic behavior on the macroscopic and microscopic scale, explains the microscopic isotropic response of the obtained material.

Nevertheless, probing for the response of the macroscopic sample to the external magnetic field of a strong permanent magnet resulted in a dependency of the attraction on the intrinsic anisotropic structure of the wooden cube. Approaching the magnetized sample with the field lines of the permanent magnet in parallel to the fiber direction resulted in a direct attraction. In contrast, an approach with the field lines perpendicular to the fiber direction resulted in flipping of the magnetized sample and subsequent alignment of the fibers in the external magnetic field (Fig. 6b). Therefore, although the deposited magnetic phase shows the isotropic characteristic and dominates the microscopic behavior of the resulting hybrid material, the anisotropy of the macroscopic hierarchical structure of the wood is dominating the response of the composite on the macroscopic scale.

\section{Conclusion}

A microwave-assisted thermal decomposition synthesis for superparamagnetic nanoparticles (SPIONs) has been used as model protocol of an efficient approach for the hybridization and functionalization of wood. We could show that an integration of the nanoparticles deep into the wood cell wall structure can be achieved, which was confirmed by confocal Raman spectroscopy imaging.

The fine dispersion and uniform distribution of SPIONs in all anatomical regions are key factors to achieve a homogeneous property distribution even on the macroscopic scale. The integration of the nanostructures into the compound middle lamella, the interconnecting system running through the whole macroscopic volume, is of high relevance for the development of interconnected functional phases and a transfer of the microscopic properties to the macroscopic level. The high flexibility and broad applicability of microwave-assisted synthesis for the preparation of nanosized materials of various composition, enable a highly versatile and energy efficient strategy for the future integration of different functionalities into the cell wall structure of wood.

Moreover, control over the content and distribution of the particles could be potentially established by tailoring the amount of accessible hydroxyl groups in wood, which act as nucleation sites.

The obtained particle load, although limited to approximately $5 \mathrm{wt} \%$ of the total material, is sufficient to obtain a good response to external magnetic fields. Due to the moderate temperatures of the microwave approach, instead of using common nanoparticle synthesis by co-precipitation of $\mathrm{FeCl}$ and $\mathrm{FeCl}$ at room temperature, a good degree of crystallinity of the particles and consequently high magnetization values are realized.

The functionalized wood samples showed anisotropic behaviour in their macroscopic response to external magnetic fields along the wood fibers of the composite material, while on the microscopic scale no anisotropy of the formed material was observed. 


\section{Conflicts of interest}

There are no conflicts to declare.

\section{Acknowledgements}

This work was financially supported by the HINT Network (COST Action MP 1202), EU FP7 MultiBioPro Project, ETH Zurich, the Spanish Ministry of Economy (Juan de la Cierva Postdoctoral Fellow FJCl-2014-21407 to AM-M, MAT2015-64442-R and SEV-2015-0496 projects, co-funded with European Social Funds). We would like to acknowledge as well the Center for Research in Agricultural Genomics (CRAG) - UAB Barcelona for technical support.

\section{References}

1 C. Sanchez, H. Arribart and M. M. G. Guille, Nature Materials, 2005, 4, 277-288.

2 J. W. C. Dunlop and P. Fratzl, in Annual Review of Materials Research, Vol 40, eds. D. R. Clarke, M. Ruhle and F. Zok, 2010, pp. 1-24.

3 U. G. K. Wegst, H. Bai, E. Saiz, A. P. Tomsia and R. O. Ritchie, Nature Materials, 2015, 14, 23-36.

4 J. K. Guest and J. H. Prevost, International Journal of Solids and Structures, 2006, 43, 7028-7047.

5 S. Weiner and P. M. Dove, in Biomineralization, eds. P. M. Dove, J. J. DeYoreo and S. Weiner, 2003, pp. 1-29.

6 E. Cabane, T. Keplinger, V. Merk, P. Hass and I. Burgert, ChemSusChem, 2014, 7, 1020-1025.

7 M. A. Ermeydan, E. Cabane, N. Gierlinger, J. Koetz and I. Burgert, RSC Advances, 2014, 4, 1298112988.

8 Q. L. Fu, L. Medina, Y. Y. Li, F. Carosio, A. Hajian and L. A. Berglund, ACS Applied Materials \& Interfaces, 2017, 9, 36154-36163.

9 Y. Y. Li, Q. L. Fu, R. Rojas, M. Yan, M. Lawoko and L. Berglund, ChemSusChem, 2017, 10, 3445-3451.

10 Y. Y. Li, Q. L. Fu, S. Yu, M. Yan and L. Berglund, Biomacromolecules, 2016, 17, 1358-1364.

11 Y. Y. Li, S. Yu, J. G. C. Veinot, J. Linnros, L. Berglund and I. Sychugov, Advanced Optical Materials, 2017, 5.

V. Merk, M. Chanana, N. Gierlinger, A. M. Hirt and I. Burgert, ACS Applied Materials \& Interfaces, 2014, 6, 9760-9767.

B. Unger, M. Bucker, S. Reinsch and T. Hubert, Wood Science and Technology, 2013, 47, 83-104. M. W. Zhu, J. W. Song, T. Li, A. Gong, Y. B. Wang, J. Q. Dai, Y. G. Yao, W. Luo, D. Henderson and L. B. Hu, Advanced Materials, 2016, 28, 5181-+.

15 T. Keplinger, E. Cabane, J. K. Berg, J. S. Segmehl, P. Bock and I. Burgert, Advanced Materials Interfaces, 2016, 3.

S. Y. Lv, F. Fu, S. Q. Wang, J. D. Huang and L. Hu, RSC Advances, 2015, 5, 2813-2818.

S. Trey, R. T. Olsson, V. Strom, L. Berglund and M. Johansson, RSC Advances, 2014, 4, 35678-35685.

V. Merk, J. K. Berg, C. Krywka and I. Burgert, Crystal Growth \& Design, 2017, 17, 677-684.

O. Weigenand, H. Militz, P. Tingaut, G. Sebe, B. de Jeso and C. Mai, Holzforschung, 2007, 61, 51-59.

N. Pinna and M. Niederberger, Angewandte Chemie-International Edition, 2008, 47, 5292-5304.

N. Kranzlin, S. Ellenbroek, D. Duran-Martin and M. Niederberger, Angewandte Chemie-International Edition, 2012, 51, 4743-4746.

22 S. M. Yu, J. A. Hachtel, M. F. Chisholm, S. T. Pantelides, A. Laromaine and A. Roig, Nanoscale, 2015, 7, 14039-14046.

23 M. Zeng, A. Laromaine, W. Q. Feng, P. A. Levkin and A. Roig, Journal of Materials Chemistry C, 2014, 2, 6312-6318. 
Z. G. Ding, S. Q. Shi, H. L. Zhang and L. P. Cai, Composites Part B-Engineering, 2015, 78, 266-271.

W. Gan, L. Gao, S. Xiao, R. Gao, W. Zhang, J. Li and X. Zhan, Advanced Materials Interfaces, $2017,4$.

H. Oka and H. Fujita, leee Transactions on Magnetics, 1999, 35, 3520-3522.

H. Oka, H. Hamano and S. Chiba, Journal of Magnetism and Magnetic Materials, 2004, 272, E1693E1694.

H. Oka, A. Hojo, K. Seki and T. Takashiba, Journal of Magnetism and Magnetic Materials, 2002, 239, 617-619. Chemistry C, 2012, 116, 15108-15116.

30 D. Fengel, A. G. Siemens and H. Wolfsgruber, Holz als Roh-und Werkstoff, 1971, 29, 67-+.

31 I. Chourpa, L. Douziech-Eyrolles, L. Ngaboni-Okassa, J. F. Fouquenet, S. Cohen-Jonathan, M. Souce, H. Marchais and P. Dubois, Analyst, 2005, 130, 1395-1403.

32 N. Gierlinger and M. Schwanninger, Plant Physiology, 2006, 140, 1246-1254.

33 D. L. A. deFaria, S. V. Silva and M. T. deOliveira, Journal of Raman Spectroscopy, 1997, 28, 873-878.

34 O. N. Shebanova and P. Lazor, Journal of Solid State Chemistry, 2003, 174, 424-430.

35 R. J. Thibeau, C. W. Brown and R. H. Heidersbach, Applied Spectroscopy, 1978, 32, 532-535.

36

W. B. White and Deangeli.Ba, Spectrochimica Acta Part a-Molecular Spectroscopy, 1967, A 23, 985-\&. 